山्山FANÇAISE

$>\mathrm{DE}$

$\stackrel{1}{\simeq}$ PÉDAGOGIE

\section{Revue française de pédagogie}

Recherches en éducation

$205 \mid 2018$

Instruction(s) en famille. Explorations sociologiques d'un phénomène émergent

\title{
FABRE Michel \& CLAVIER Loïc (dir.). Le religieux sans la religion. Vivre et éduquer sans absolu? (1850-1950)
}

Mont-Saint-Aignan : Presses universitaires de Rouen et du Havre, 2019, $244 \mathrm{p}$.

François Jacquet-Francillon

\section{OpenEdition \\ Journals}

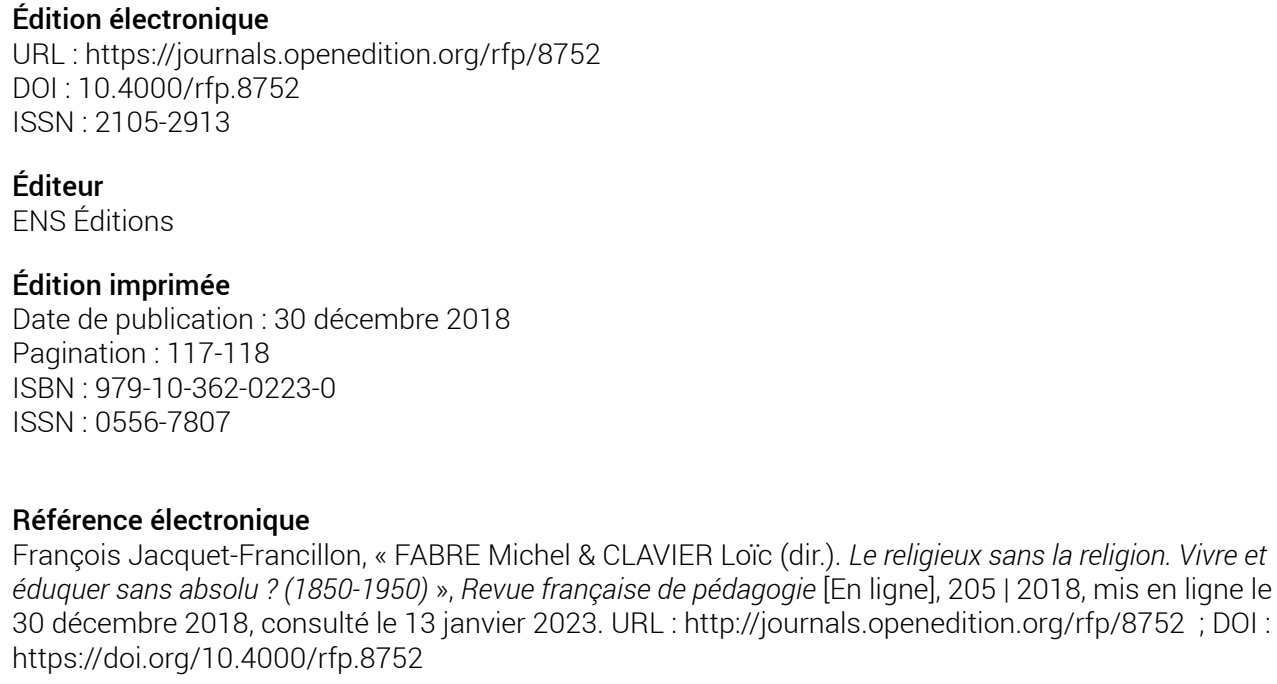
éduquer sans absolu? (1850-1950) », Revue française de pédagogie [En ligne], 205 | 2018, mis en ligne le 30 décembre 2018, consulté le 13 janvier 2023. URL : http://journals.openedition.org/rfp/8752 ; DOI : https://doi.org/10.4000/rfp.8752

Ce document a été généré automatiquement le 13 janvier 2023.

Tous droits réservés 


\section{FABRE Michel \& CLAVIER Loïc (dir.). Le religieux sans la religion. Vivre et éduquer sans absolu? (1850-1950)}

Mont-Saint-Aignan : Presses universitaires de Rouen et du Havre, 2019, $244 \mathrm{p}$.

François Jacquet-Francillon

\section{RÉFÉRENCE}

FABRE Michel \& CLAVIER Loïc (dir.). Le religieux sans la religion. Vivre et éduquer sans absolu ? (1850-1950). Mont-Saint-Aignan : Presses universitaires de Rouen et du Havre, $2019,244 \mathrm{p}$.

Cet ouvrage, publié dans la collection que dirige Marie-Louise Martinez ( $«$ Penser les valeurs en éducation et en formation »), comporte une série de contributions visant à interroger ce qu'on pourrait appeler la pensée ou la culture laïque (expression que n'utilisent pas les auteurs), en entendant par là cet ensemble d'élaborations ou de réélaborations intellectuelles qui, à la fin du xix $x^{e}$ siècle et au début $d u x^{e}$, souvent dans le contexte de l'instauration française de la laïcité de l'école primaire, ont tenté de dessiner un avenir et un présent du monde social « sécularisé » voire même dispensé de toute référence religieuse dogmatique. Sont en l'occurrence analysés les apports des théories, disons même des doctrines politiques et philosophiques de Comte (par Alain Kerlan), de Durkheim (par Marie-Louise Martinez), de Weber (par Céline Chauvigné), de Renan (par Alfred-Romuald Gambou), de Jules Barni (par Pierre Billouet), d'Edgar Quinet (par Jean-Marc Lamarre), de Ferdinand Buisson (par Loïc Clavier), de John Dewey (par Michel Fabre), de Tolstoï (par Loïc Clavier), de Jean-Marie Guyau (par Michel Fabre), sans oublier, beaucoup moins connu chez nous mais tout aussi intéressant, le chinois Kang Youwei (par Roland Depierre). 
2 L'ouvrage se compose de trois parties: d'abord une partie où sont ressaisies les conditions dans lesquelles le phénomène religieux a pu faire l'objet d'une approche rationnelle ou scientifique (de Comte à Durkheim et Weber); ensuite une partie (où l'on voit apparaitre les œuvres de Barni, de Quinet ou de Buisson) consacrée aux conflits entre religion et politique, conflits et autres tensions débouchant sur la séparation de l'Église et de l'État; enfin, sous un angle peut-être pas biographique mais plus singularisant, une partie permettant de suivre les trajets singuliers de la " conversion " ou de la « déconversion» de certains auteurs (comme Tolstoï), notamment quand ces trajets mènent à l'athéisme ou à une « irreligion » résolument affirmée (c'est le cas de Jean-Marie Guyau, prônant "L'irreligion de l'avenir »).

3 Au crédit de ces analyses, on peut mettre d'abord le fait qu'elles traitent les principaux représentants français de ces courants de pensée, représentants assez présents dans nos mémoires mais trop peu étudiés assurément ou, du moins, dont nous connaissons mal les interprétations du phénomène religieux. Grâce à ces études, et c'est le premier mérite de ce livre, nous pouvons donc renouer avec des auteurs et un questionnement à bien des égards fondateurs de la modernité quoique souvent sous-estimés par nos contemporains (à part Durkheim et Weber... peut-être!). Une autre réussite de l'ouvrage tient à ce qu'il s'efforce de dégager un fonds commun de préoccupations et de thématiques mais sans gommer les différences d'appréciation et même les divergences, notamment politiques, entre les auteurs analysés.

$4 \quad$ Un autre constat est essentiel de ce point de vue : la plupart des auteurs étudiés ici ont développé une pensée typique de la sécularisation, une pensée et une conviction de l'épuisement de la transcendance et du retrait hors de l'espace de la cité des pratiques rituelles religieuses; mais, d'un autre côté, étant bien davantage anticléricaux qu'irreligieux, ils n'ont pas voulu que la religion s'absente entièrement de la vie collective et ne gouverne plus le destin des sociétés. C'est ainsi que Comte attendait et préparait l'instauration d'un nouveau pouvoir spirituel à la place de l'Église et du Clergé ; c'est ainsi que Renan, lui aussi, appelait de ses vœux une autre religion que le christianisme dont il avait voulu retracer la genèse ; c'est ainsi, encore, que Buisson parlait de «foi laïque » ou qu'Edgar Quinet parlait d'une « institution religieuse de la société »- toutes choses qui justifient bien le titre de l'ouvrage dont il est ici question : «Le religieux sans la religion »... En d'autres termes, nous aurions affaire à du religieux mais en un sens nouveau, à savoir du religieux sans révélation ni théologie.

5 C'est dire que les grands auteurs laïcs ont imaginé non pas une sortie globale et définitive de la religion (si tant est que "sortir " signifie quitter et non pas conserver un héritage en l'absence du légataire, comme on dit qu'on est sorti d'une grande école pour annoncer qu'on reste ad vitam aeternam dépendant de la formation qu'on y a reçue), non pas une sortie de la religion disais-je, mais une sorte d'épiphanie de la spiritualité posée comme une nécessité pour l'organisation de la vie commune. Quel sens les auteurs concernés donnent-ils dans ce cas à une telle spiritualité ? C'est justement ce que ce livre nous apprend. Disons que le spirituel se rapporte à la morale ; disons même plus clairement que le spirituel tient purement et simplement dans une morale c'est-à-dire dans un ensemble de commandements ou d'obligations que les individus se donnent et qu'ils respectent pour le maintien de la communauté humaine à laquelle ils appartiennent.

6 Or la morale et l'idéal (l'« absolu » dit le sous-titre de l'ouvrage), qui sont peut-être une nouvelle modalité du sacré (notion utilisée à tort et à travers aujourd'hui, et assez peu 
abordée dans ce livre), sont en général conçus comme des produits de la raison. Ainsi pouvons-nous comprendre que ces auteurs, à des titres et à des degrés divers, manifestent tous, peu ou prou, une conviction rationaliste. Pour eux, il y a et il doit y avoir du religieux en l'absence de religion et de dieu; mais ce religieux, cette spiritualité, d'une part doit délivrer une morale, et d'autre part une morale postulée par la raison individuelle. Proposition vérifiable chez Comte, chez Durkheim (qui parle d'un culte - moral- de la personne humaine), de même que chez Jules Barni (traducteur et introducteur de Kant), chez Renan, Quinet, Buisson ou encore Dewey aux États-Unis. Certes, l'idée même de la raison ne fait pas l'objet d'un consensus de définition. Mais chez ces auteurs on trouve toujours exprimée la conviction qu'est possible et hautement souhaitable une religion qu'on pourrait dire «naturelle » (sur un modèle issu $d u x{ }^{\prime} I^{e}$ siècle), en tout cas une religion immanente essentiellement porteuse d'une morale et à laquelle tout individu est censé accéder par l'exercice de ses propres facultés rationnelles.

7 S'il fallait citer sur ce plan un seul auteur à la fois très représentatif et presque oublié, ce serait sans doute Jean-Marie Guyau, auquel Michel Fabre consacre ici une étude spéciale, fort bienvenue. J.-M. Guyau, auteur de l'Esquisse d'une morale sans obligation ni sanction (1885), est en effet celui qui a le plus systématiquement (et consciemment) noué le lien entre une critique de la religion révélée et la volonté de conserver une sorte d'intuition religieuse originelle, tout comme le lien de cette intuition avec une association humaine possiblement établie sur des bases solides (des bases vitales dans la pensée de Guyau, inspiré par Darwin et la théorie de l'évolution).

Rendons-nous attentifs, par conséquent, à la synthèse de ces problématiques, clairement présentée en conclusion de l'ouvrage par Anne Vézier. D'après cette dernière, et on ne peut que la suivre, la culture de la sécularisation développée depuis la fin $\mathrm{du} \mathrm{xIX}^{\mathrm{e}}$ siècle est structurée par trois schèmes, chacun décrivant une modalité particulière du processus de sécularisation, très complexe et, finalement, très nuancé. En premier lieu un schème de retour à - je préférerais dire: de préservation d'un sentiment religieux. Ensuite un schème de transfert du sacré vers la réalité de l'Humanité (on pourrait discuter de cette idée d'un «transfert de sacralité », notion très appréciée par les historiens, mais l'identification de l'Humanité comme figure d'autorité morale substituée à Dieu est très fidèle à l'enseignement positiviste - pour ne citer que lui). Enfin, logiquement, un schème de dépassement des religions, un tel dépassement ouvrant une perspective d'émancipation - qui d'ailleurs ne peut manquer d'avoir de puissants effets sur les conceptions de l'éducation associées.

9 Faut-il, au bout du compte, tabler sur « une morale, une spiritualité ou une culture sans la religion" (Anne Vézier, p. 213) ? La voie semble ouverte... Cette étude a ceci de précieux qu'elle montre bien les moyens de l'emprunter. Mais jusqu'où? Telle est la question qui demeure, pour notre actualité. 